\title{
Inflammation, Impaired Motility, and Permeability in a Guinea Pig Model of Postoperative Ileus
}

\author{
Yoo Jin Lee, ${ }^{1}$ Zahid Hussain, ${ }^{2}$ Cheal Wung Huh, ${ }^{2}$ Young Ju Lee, ${ }^{2}$ and Hyojin Park ${ }^{2 *}$ \\ ${ }^{I}$ Department of Internal Medicine, Keimyung University School of Medicine, Daegu, Korea; and ${ }^{2}$ Department of Internal Medicine, Gangnam \\ Severance Hospital, Yonsei University College of Medicine, Seoul, Korea
}

\section{Background/Aims}

Postoperative ileus (POI) is characterized by impaired propulsive function of the gastrointestinal tract after surgery. Although inflammation is considered to be an important pathogenesis of POI, significant data are lacking. We aim to correlate the recovery time of postoperative dysmotility with that of inflammation and mucosal permeability.

\section{Methods}

An experimental POI model of guinea pig was used. Contractile activity of the circular muscle of the stomach, jejunum, ileum, and proximal colon was measured through a tissue bath study. Inflammatory cells were counted, and the expression of calprotectin and tryptase were analyzed. The expression of protease-activated receptor 2 (PAR-2), claudin-1, and claudin-2 were analyzed with immunofluorescence.

\section{Results}

The small bowel and colon showed decreased contractile amplitude in the POI groups compared to control. In contrast to the colon, the contractile amplitude of the small bowel significantly recovered in the POI group at 6 hours after the operation compared to the control group. Inflammation was highly significant in the POI groups compared to the control and sham groups, especially in the colon. Immunofluorescence showed increased PAR-2 expression in the POI groups compared to sham. The decreased claudin-1 expression and increased claudin-2 expression may suggest increased mucosal permeability of the small bowel and colon in the POI groups.

\section{Conclusions}

Increased inflammation and mucosal permeability may play an important role in the differential recovery stages in POI. These data may provide further insights into the pathophysiology and potential new therapeutic prospects of $\mathrm{POI}$.

(J Neurogastroenterol Motil 2018;24:147-158)

\section{Key Words}

Ileus; Inflammation; Permeability

Received: February 10, 2017 Revised: May 23, 2017 Accepted: August 16, 2017

(a) This is an Open Access article distributed under the terms of the Creative Commons Attribution Non-Commercial License (http://creativecommons. org/licenses/by-nc/4.0) which permits unrestricted non-commercial use, distribution, and reproduction in any medium, provided the original work is properly cited.

${ }^{*}$ Correspondence: Hyojin Park, MD, PhD

Department of Internal Medicine, Gangnam Severance Hospital, Yonsei University College of Medicine, 211 Eonju-ro, Gangnam-gu, Seoul 06273, Korea

Tel: +82-2-3463-3882, Fax: +82-2-3463-3882, E-mail: HJPARK21@yuhs.ac 


\section{Introduction}

Postoperative ileus (POI) is the transient cessation of coordinated bowel motility after abdominal surgical intervention in most patients. ${ }^{1-3}$ POI lasts approximately 3-5 days with clinical features of abdominal distension, bloating, pain, nausea, vomiting, and lack of bowel sounds. ${ }^{4}$ The prevention of POI is absolutely important, as ileus has a negative impact on the quality of life and increases the length of hospital stay, morbidity, and socio-economic costs. ${ }^{5-7}$ However, no satisfactory treatment strategy to prevent or treat POI has been established yet. The mechanism of POI is suggested to be complex and multifactorial, and has been broadly categorized into 5 main theories, including the activation of inhibitory spinal and inhibitory sympathetic reflexes, anesthetics, humoral agents, and inflammation. ${ }^{8}$ Abdominal surgery triggers 2 phases. The first phase is the neurogenic phase, which is short and ends early after the surgery. The second is the inflammatory phase, which starts 3-4 hours after surgery and persists much longer. ${ }^{4}$ Even the gentlest surgical handling of the small intestine consequently activates a typical acute inflammatory cascade within the muscle layers of the intestinal wall. ${ }^{9,10} \mathrm{~A}$ characteristic of this local inflammation is the activation of the resident macrophages that lie as sentinels within the enteric muscularis. ${ }^{11}$ This activation leads to phosphorylation of transcription factors, resulting in the subsequent induction of genes and release of cytokines, chemokines, and kinetically active substances such as nitric oxide and prostaglandins. ${ }^{12}$ This local inflammatory milieu participates in the upregulation of adhesion molecules, which recruit degranulating leukocytes into the muscularis. ${ }^{11,12}$

According to the current understanding, the inflammatory phase plays a significant role in the genesis and maintenance of gastrointestinal (GI) dysmotility after surgery, and is expected to be an important target for the future treatment of POI. However, there is still limited evidence regarding the association between inflammation and the recovery time from ileus in relation to different GI organs. Consequently, we hypothesized that the changes in gut paracellular permeability may affect inflammation, and hence intestinal transit, in POI. Therefore, the aim of this study was to investigate the changes in inflammation and paracellular permeability in relation to the pathogenesis of POI.

\section{Materials and Methods}

\section{Preparation and Handling of Different Groups of Animals}

Adult male guinea pigs (Orient Bio Inc, Seoul, Korea) weighing 250-350 g were individually kept and acclimatized in environmentally controlled conditions $\left(21 \pm 1^{\circ} \mathrm{C}, 50 \pm 10 \%\right.$ humidity, and 12-hour light/dark cycle commencing at 7 AM). A standard guinea pig diet and water were provided ad libitum. The guinea pigs were subjected to a 24-hour fast with free access to water before the experiments. ${ }^{13-15}$ The animals assigned as the control group were administered anesthesia only. The sham group underwent laparotomy under anesthesia without manipulation of the cecum. The POI model received laparotomy followed by evisceration and gentle manipulation of the cecum by using a wet gauge for 60 seconds, and then closure with sutures. All experiments were conducted in agreement with the guidelines of the Ethical Animal Research Committee of the University of the Department of Laboratory Animal Medicine, Medical Research Center, Yonsei University College of Medicine (2013-0343).

\section{Measurement of Contractile Activity Through a Tissue Bath Study}

We delicately peeled off the mucosa and submucosa of the stomach, jejunum, ileum, and proximal colon. Exposed circular muscle strips from each segment were cut into segments of 3-mm width and 10-mm length. After flush cleaning with KrebsHenseleit solution, muscle strips were pinned and connected to tension transducers (BIOPAC TSD 105; BIOPAC System Inc, Santa Barbara, CA, USA). Then, the isometric tension was documented on physiography (Grass, Quincy, MA, USA). ${ }^{16}$ A baseline $1 \mathrm{~g}$ of tension was loaded onto the circular muscle strip during the 60 minutes equilibrium period. ${ }^{17,18}$ Electrical field stimulation was conducted to estimate contraction of each organ using a stimulator (Grass S88; Astro-Med, West Warwick, RI, USA). Based on the methods used in other studies, the detailed conditions of electrical field stimulation were as follows: antrum ( 1 millisecond pulse duration, $80 \mathrm{~V}$, for 10 seconds); jejunum (1 millisecond pulse duration, $60 \mathrm{~V}$, for 10 seconds); ileum (0.5 milliseconds pulse duration, $60 \mathrm{~V}$, for 10 seconds); and proximal colon ( 0.5 milliseconds pulse duration, $60 \mathrm{~V}$, for 10 seconds) in a stepwise-increment of frequency (2, 4, 8 , and $16 \mathrm{~Hz}$ ). Ten stimulations were administered at 1-minute intervals, and the mean amplitude was measured. Data are expressed 
as percentages of the change compared to baseline.

\section{Measurement of the Degree of Inflammation}

Histologic sections obtained from the muscle coat of the stomach, jejunum, ileum, and proximal colon at 3 hours and 6 hours after the operation were fixed in $10 \%$ formalin solution neutral buffered and then embedded in paraffin. Each embedded section was sliced into $4 \mu \mathrm{m}$ thickness and stained with hematoxylin and eosin. We employed a semi-quantitative scoring system to compare the degree of inflammation between the sham and POI groups. ${ }^{15,19}$

\section{Calprotectin and Tryptase Expression}

The expression of calprotectin and tryptase was studied by using immunohistochemistry. The paraffin-embedded histologic tissue sections of the stomach, jejunum, ileum, and colon segments
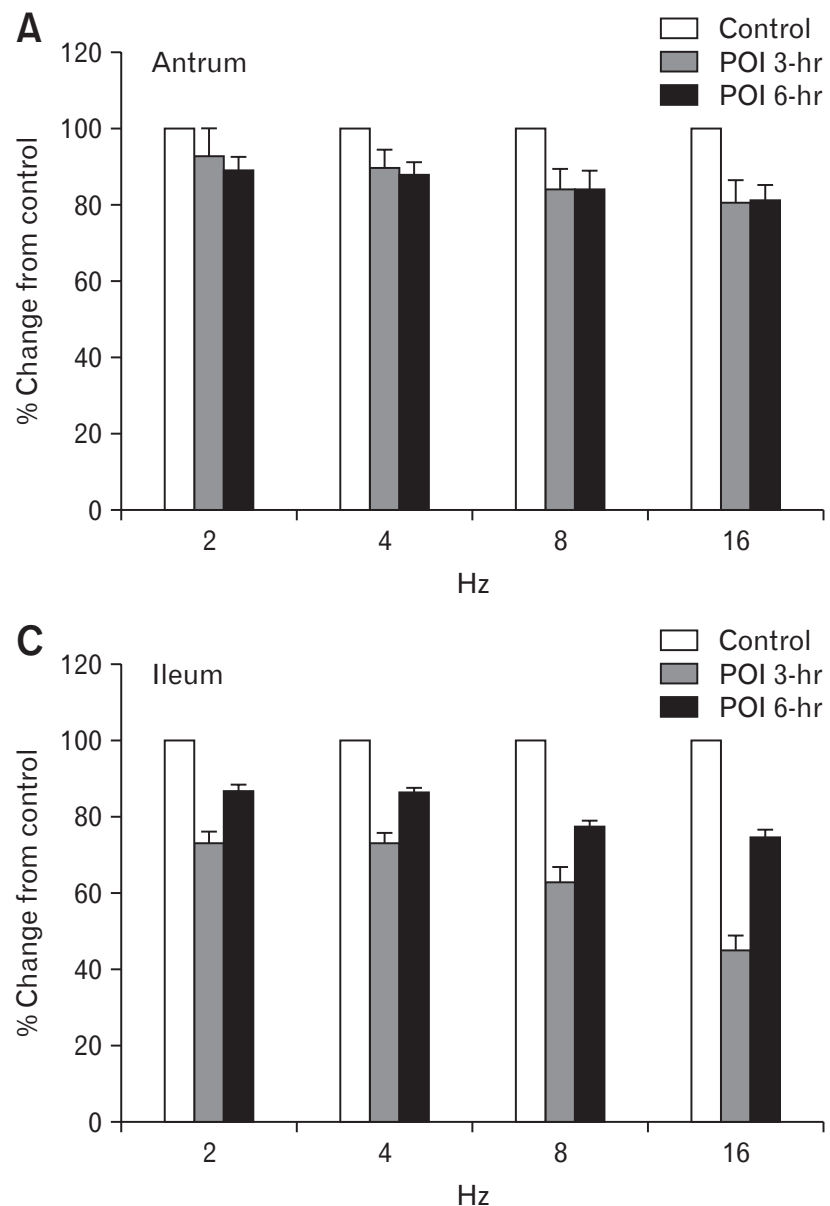

were deparaffinized. Endogenous peroxidase activity was blocked by incubating the tissue sections in $3 \%$ hydrogen peroxide for 10 minutes. The tissue section was then incubated with primary antibodies, anti-calprotectin (1:200; Abcam, Cambridge, MA, USA) and anti-mast cell tryptase (1:500; Abcam), followed by the secondary antibody anti-mouse IgG (1:1000; Santa Cruz Biotechnology, Dallas, TX, USA). Then, the sections were incubated with streptavidin-horseradish peroxide for 30 minutes followed by treatment with AB-peroxidase solution and counterstaining with hematoxylin. Positively stained cells were then examined under a fluorescence microscope (Zeiss Axio Imager Z1; Carl Zeiss, Jena, Germany).

\section{Expression of Protease-activated Receptor 2, Claudin-1, and Claudin-2}

The expression of protease-activated receptor 2 (PAR-2), clau-
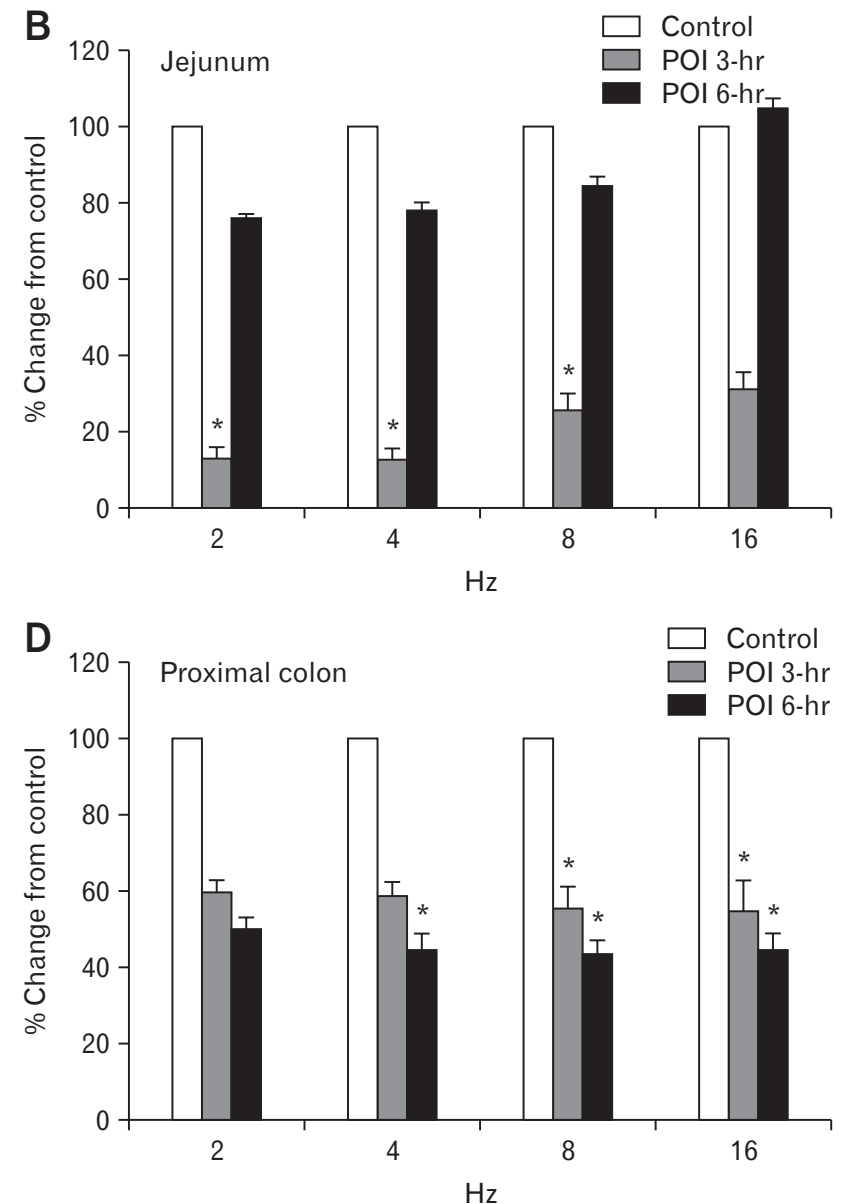

Figure 1. The amplitude of contraction in the postoperative ileus (POI) groups and controls in different gastrointestinal organs: (A) antrum, (B) jejunum, (C) ileum, and (D) proximal colon. Data are expressed as the percentage change compared to baseline. Bars indicate mean \pm SEM ( $\mathrm{n}=$ 5 per group). ${ }^{*} P<0.05$ was considered to indicate statistical significance compared to controls. POI 3-hr, POI group at 3 hours after the operation; POI 6-hr, POI group at 6 hours after the operation. 

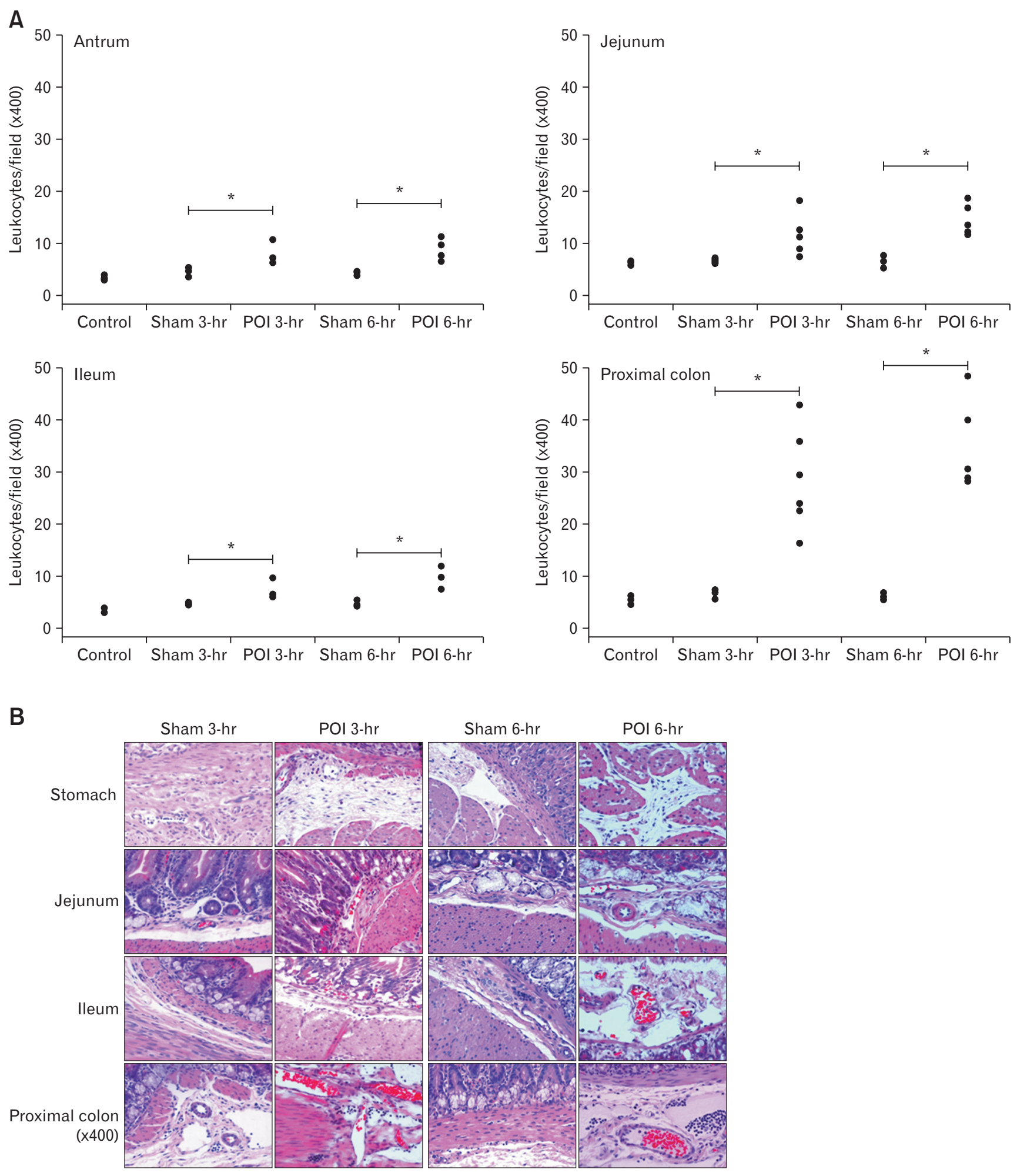

Figure 2. The degree of inflammation in the postoperative ileus (POI) and sham groups. (A) Semi-quantitative analysis of inflammatory cells was done by counting the leukocytes per field under bright field microscopy. Scatter dot plots showed significantly increased expression of inflammation in POI groups compared to sham groups (control, $\mathrm{n}=4$; sham, $\mathrm{n}=4$; POI 3-hr, $\mathrm{n}=6$; POI 6 - $\mathrm{hr}, \mathrm{n}=5$ ). ${ }^{*} \mathrm{P}<0.05$ was considered to indicate statistical significance compared to the sham groups. (B) Representative hematoxylin and eosin images of the POI and sham groups $(\times 400$ magnification). 

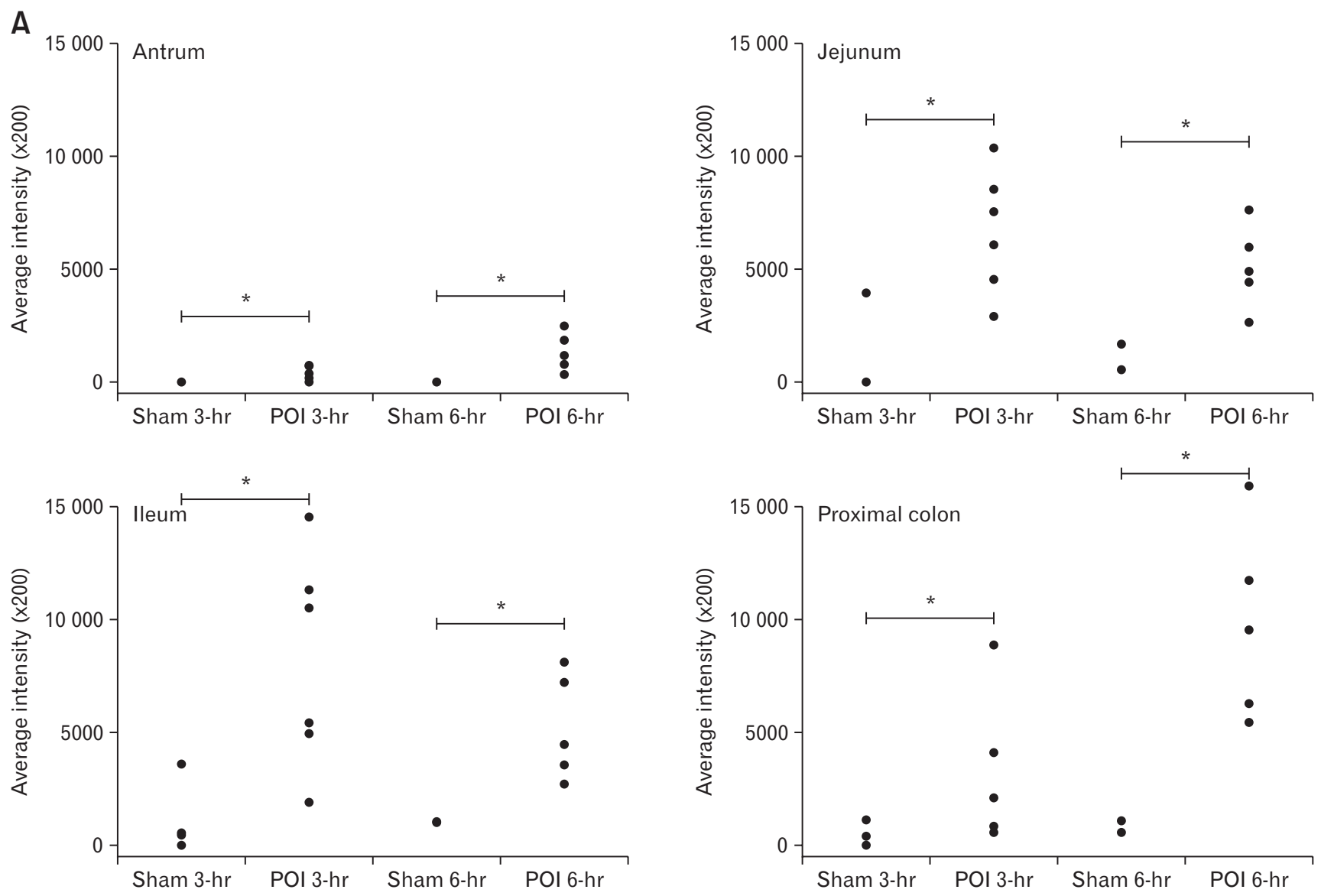

B
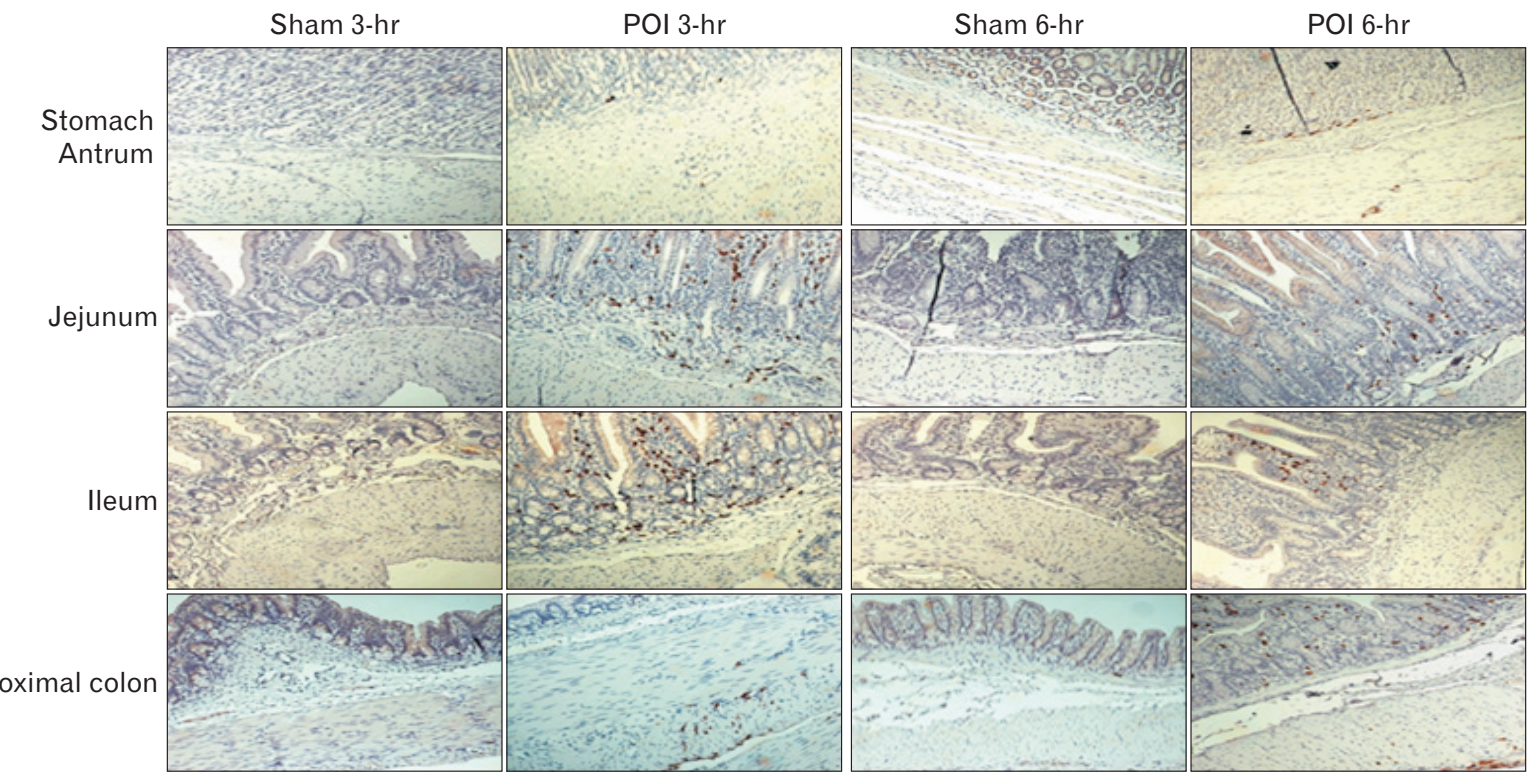

Figure 3. Immunohistochemical staining of calprotectin in the postoperative ileus (POI) and sham groups. (A) The average intensity of calprotectin expression was analyzed by using MetaMorph microscopy automation and an image analysis software. Scatter dot plots showed significantly increased expression of calprotectin in POI groups compared to sham groups (sham 3-hr, $\mathrm{n}=4$; sham 6-hr, $\mathrm{n}=2$; POI 3-hr, $\mathrm{n}=6$; POI 6-hr, $\mathrm{n}$ $=5) .{ }^{*} P<0.05$ was considered to indicate statistical significance compared to the sham groups. (B) Representative immunohistochemical images of the POI and sham groups obtained under bright field microscopy ( $\times 200$ magnification). 


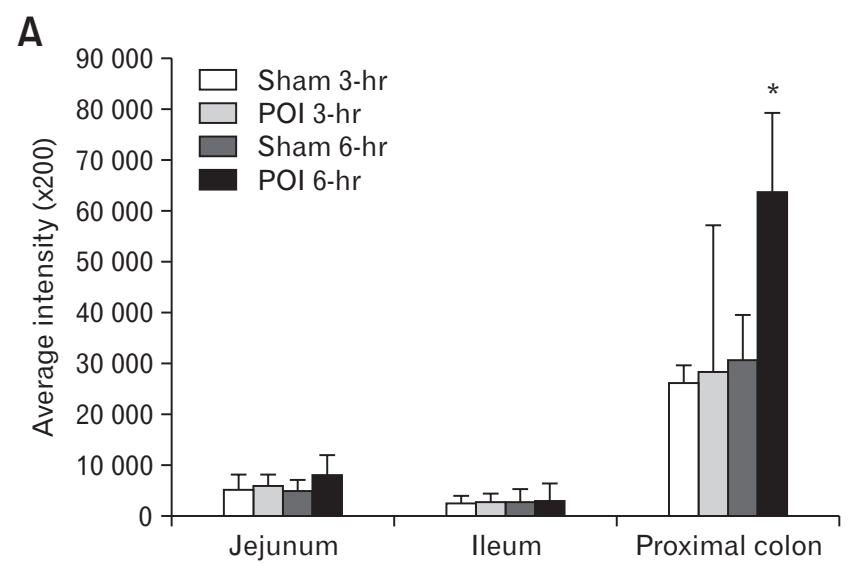

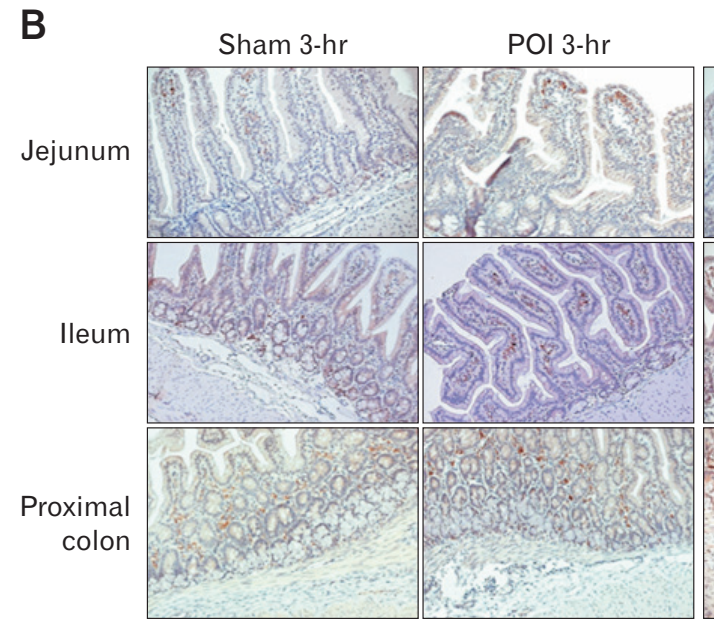

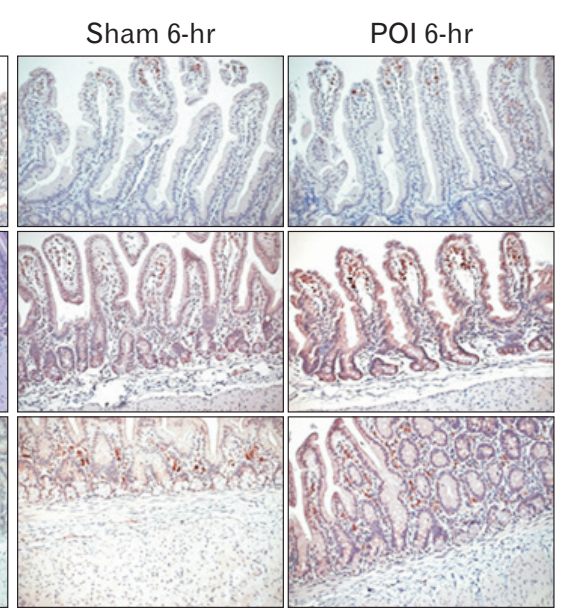

Figure 4. Immunohistochemical staining of mast cell tryptase in the postoperative ileus (POI) and sham groups. (A) The average intensity of tryptase expression was analyzed by using MetaMorph microscopy automation and an image analysis software. (B) Representative immunohistochemical images of the POI and sham groups under bright field microscopy ( $\times 200$ magnification). Data are expressed as mean \pm SEM (sham 3-hr, $\mathrm{n}=4$; sham 6-hr, $\mathrm{n}=5$; POI 3-hr, $\mathrm{n}=7$; POI 6-hr, $\mathrm{n}=7) .{ }^{*} P$ $<0.05$ was considered to indicate statistical significance compared to the sham groups. din-1, and claudin-2 was studied by using immunofluorescence. The histologic sections of the stomach, jejunum, ileum, and colon segments at 3 hours or 6 hours after the operation were fixed in $4 \%$ paraformaldehyde, embedded in paraffin, and sectioned in $4 \mu \mathrm{m}$ thick sections. After deparaffinization, rehydration, and rinsing with standard methods, the slide sections were incubated with the primary antibody rabbit anti-PAR2 (1:500; Santa Cruz Biotechnology) overnight at $4^{\circ} \mathrm{C}$, and then incubated with goat anti-rabbit IgG-fluorescein isothiocyanate (FITC) (1:200; Santa Cruz Biotechnology) for 1 hour at $37^{\circ} \mathrm{C}$. Similarly, tight junction (TJ) proteins were measured with immunofluorescence staining by using the procedure described above. Tissues were incubated with the primary antibody for claudin-1 (1:50; Invitrogen, South San Francisco, CA, USA) or claudin-2 (1:200; Invitrogen) overnight at $4^{\circ} \mathrm{C}$, then washed and incubated with the secondary antibody goat anti-rabbit IgG-FITC (1:200; Santa Cruz Biotechnology) for 30 minutes at $37^{\circ} \mathrm{C}$. The stained images were examined under a fluorescence microscope (Zeiss Axio Imager Z1; Carl Zeiss). Images analysis was performed by using MetaMorph (MDS Analytical Technologies, Sunnyvale, CA, USA).

\section{Statistical Methods}

All statistical analyses were performed by using SPSS version 18.0 (IBM Corp, Somers, NY, USA). Data are expressed as mean \pm standard error. Differences between the sham and POI groups were evaluated by using an independent $t$ test. A $P$-value of $<0.05$ was considered to indicate statistical significance.

\section{Results}

\section{Contractile Activity Among the Postoperative lleus and Control Groups}

The contractile amplitude of the stomach antrum, jejunum, ileum, and colon upon electrical stimulation was calculated by using a tissue bath study. As shown in Figure 1A and Supplementary 
Table, the change in the contractile activity of the stomach antrum was insignificant between the control and POI groups. In contrast, the contractile amplitude of the jejunum significantly decreased in the POI group at 3 hours after the operation (POI 3-hour group) compared to the control group $(P<0.05)$, whereas it recovered in the POI group at 6 hours after the operation (POI 6-hour group)

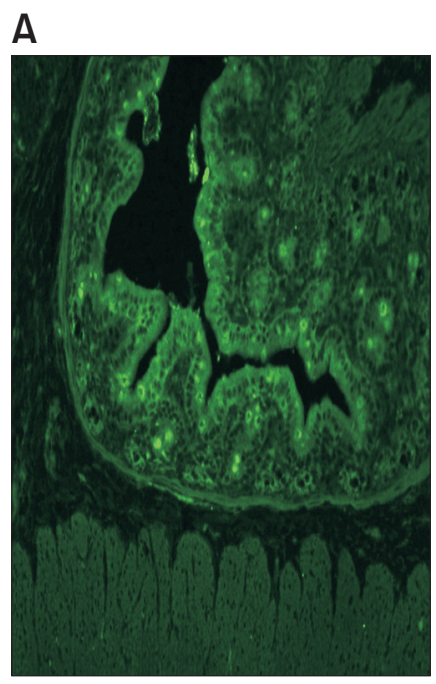

Sham 3-hr

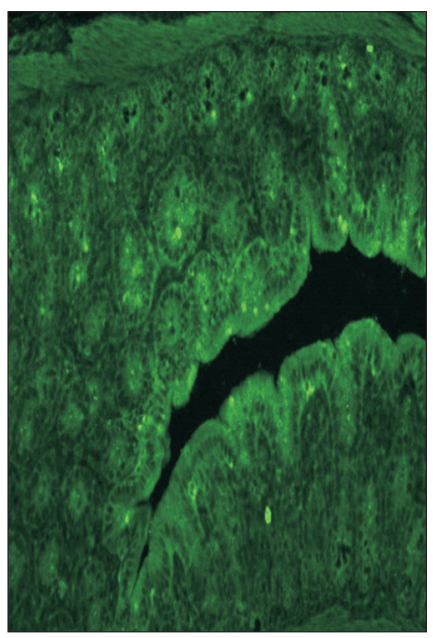

POI 3-hr

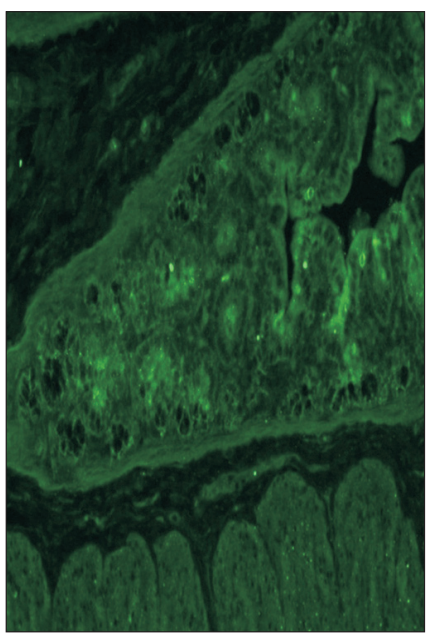

Sham 6-hr

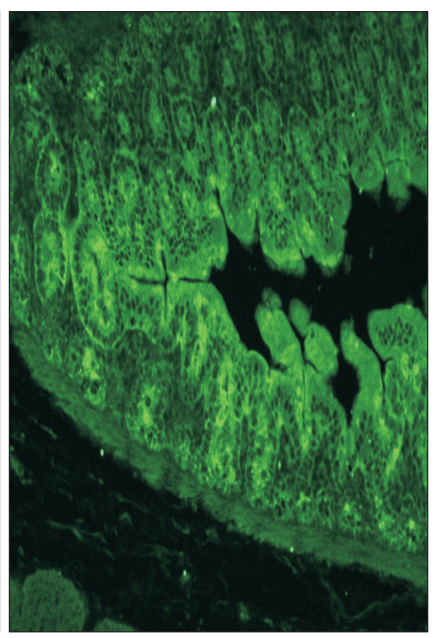

POI 6-hr

B
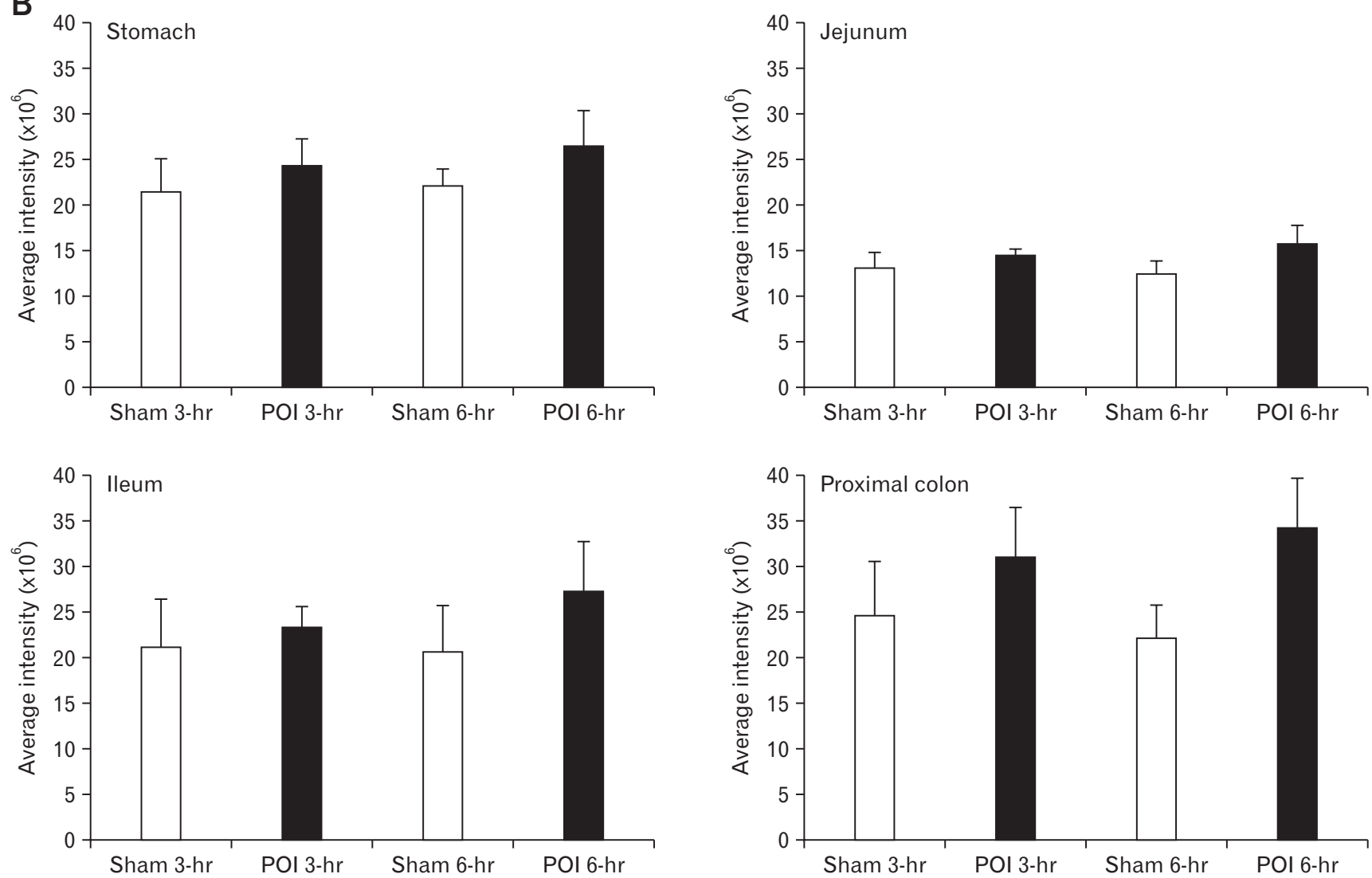

Figure 5. Protease-activated receptor 2 (PAR-2) expression on immunofluorescence staining in the postoperative ileus (POI) and sham groups. (A) Representative immunofluorescence images of PAR-2 expression in different groups of the proximal colon (B) PAR-2 expression in the stomach, jejunum, ileum, and proximal colon (sham 3-hr, $\mathrm{n}=4$; sham 6-hr, $\mathrm{n}=4$; POI 3-hr, $\mathrm{n}=6$; POI 6-hr, $\mathrm{n}=6$ ). 
A Claudin-1

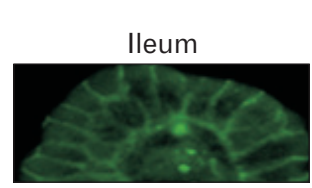

Sham 3-hr

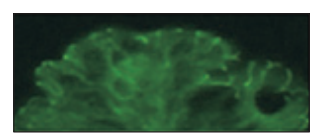

POI 3-hr

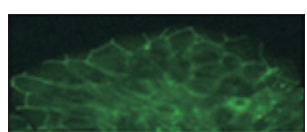

Sham 6-hr

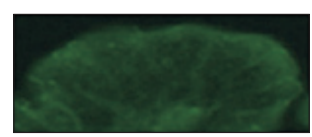

POI 6-hr

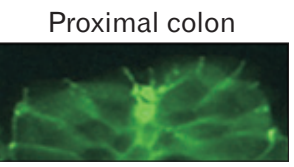

Sham 3-hr

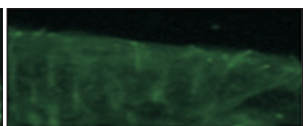

POI 3-hr

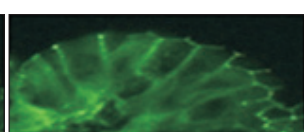

Sham 6-hr

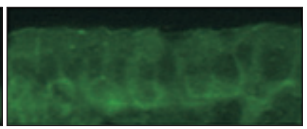

POI 6-hr

\section{B Claudin-2}
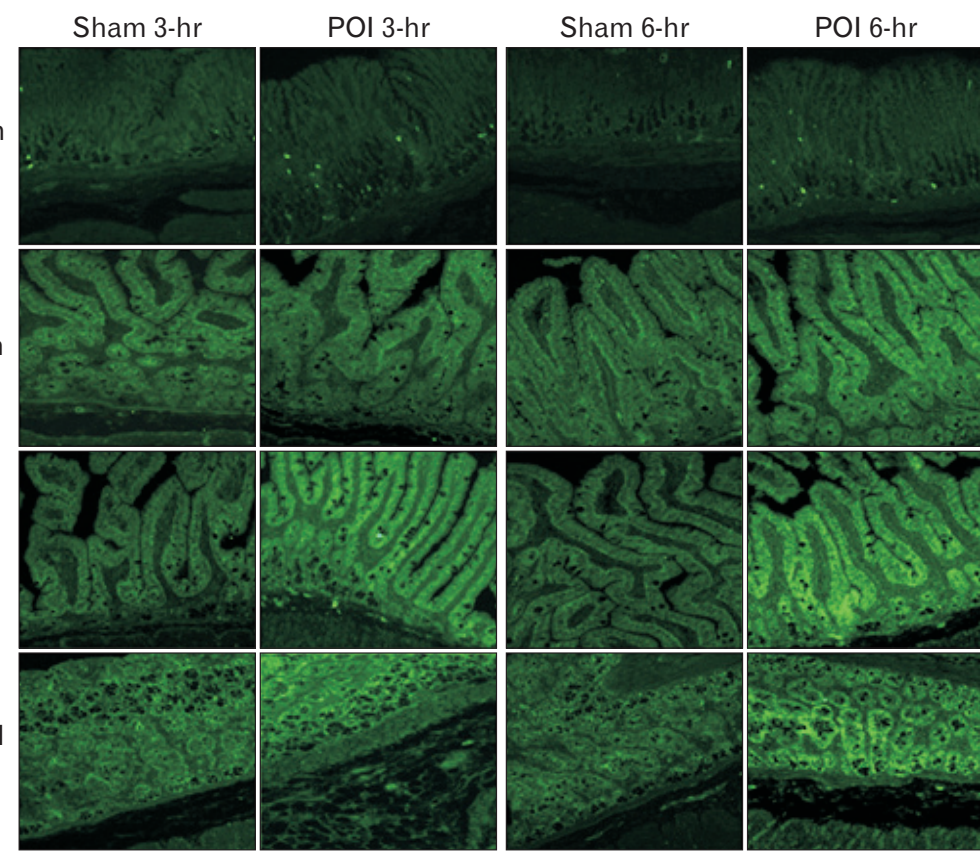

C
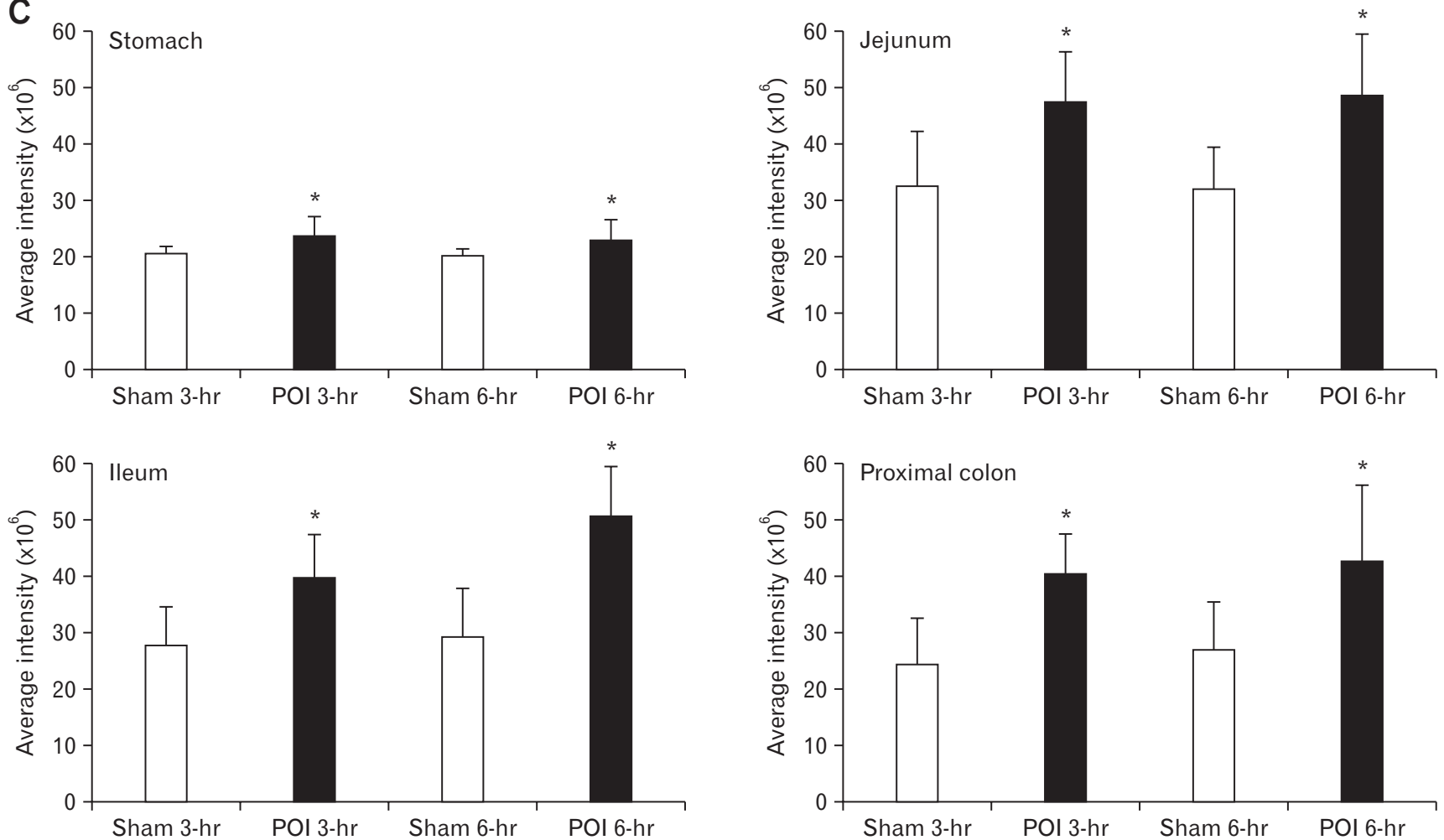

Figure 6. Expression of tight junctional proteins on immunofluorescence staining in the postoperative ileus (POI) and sham groups. (A) Representative immunohistochemical images of claudin-1 expression in different groups of the ileum and proximal colon. Decreased expression of claudin-1 was observed in the POI groups compared to the sham groups. (B) Claudin-2 expression in the stomach, jejunum, ileum, and proximal colon. (C) Claudin-2 was prominently expressed in the POI groups of the ileum and proximal colon as compared to the sham groups (sham 3-hr, $\mathrm{n}$ = 5; sham 6-hr, $\mathrm{n}=6$; POI 3-hr, $\mathrm{n}=6$; POI 6-hr, $\mathrm{n}=6$ ). 
(Fig. 1B and Supplementary Table). As compared to the control baseline status, the contractile activity in the ileum seems to decrease in the POI 3-hour group and recover in the POI 6-hour group, but there was no statistical significance (Fig. 1C and Supplementary Table). Interestingly, the contractile amplitude of the colon significantly decreased at $8 \mathrm{~Hz}$ and $16 \mathrm{~Hz}$ in the POI 3-hour group whereas the decrease appears significant at $4 \mathrm{~Hz}$ in the POI 6-hour group compared to the control group $(P<0.05)$ (Fig. 1D and Supplementary Table).

\section{Inflammatory Cell Count in the Sham and Postoperative lleus Groups}

As shown in Figure 2, there was a significant increase in the number of inflammatory cells in both the POI 3- and 6-hour groups of the antrum, jejunum, ileum, and proximal colon compared to sham groups $(P<0.05)$. Representative H\&E stain picture of leukocytes representation in the POI 6-hour group is presented in Supplementary Figure 1.

\section{Expression of Calprotectin and Mast Cell Tryptase in the Sham and Postoperative lleus Groups}

As shown in Figure 3, there was a significant increase in the expression of calprotectin in the POI 3- and 6-hour groups of the jejunum, ileum, and proximal colon compared to sham $(P<0.05)$ (Fig. 3). Representative pictures of calprotectin expression in the mucosal and muscularis externa layer in the proximal colon are presented in Supplementary Figure 2. There was an insignificant increase in mast cell tryptase expression in the POI 3- and 6-hour groups of the jejunum and ileum. However, the expression of mast cell tryptase significantly increased in the POI 6-hour group of the proximal colon $(P<0.05)$ (Fig. 4).

\section{Expression of Protease-activated Receptor 2, Claudin-1, and Claudin-2}

In the antrum, jejunum, and ileum, the expression of PAR2 was not significantly different between sham and POI groups. However, in the proximal colon, the POI 3- and 6-hour groups showed a tendency of increased expression of PAR-2 compared to sham groups, As shown in Figure 5, the POI 6-hour group showed a prominent increase in PAR-2 expression compared to the sham group, although it did not show statistical significance. To assess epithelial permeability, the expression of claudin-1 and claudin-2 was studied. The expression of claudin- 1 in the antrum and jejunum showed an insignificant change compared to the sham group. However, the expression of claudin-1 in the ileum and proximal colon showed a noticeable downregulation in the POI groups compared to the sham group (Fig. 6A and Supplementary Fig. 3A and 3B). In contrast, claudin-2 showed upregulation in the POI groups compared to the sham groups of the ileum and proximal colon (Fig. 6B).

\section{Discussion}

The aim of this study was to explore the relationship between inflammation and recovery time from POI, and to identify whether these factors are accompanied by changes in gut paracellular permeability. This study describes an experimental model of intestinal manipulation that induced the typical features of POI, such as delayed recovery of contractile activity and increased inflammation, especially in the colon. These findings were accompanied by increased expression of PAR-2 in POI models compared to sham. We also identified the changes in gut paracellular permeability through the expression of claudin-1 and claudin-2 in the POI model of guinea pigs. Our findings suggest that POI may not only result from impaired smooth muscle contractility, but inflammatory responses and paracellular permeability may also contribute to the pathophysiologic mechanism of POI.

We created the experimental model of POI at 3 hours and 6 hours after cecal manipulation, according to the results of our earlier study that showed that the degree of ileus exhibited a peak pattern at 3 hours after the procedure and recovered at 6 hours in the POI models, suggesting that 3 hours and 6 hours are the appropriate time points to estimate the early stage of POI. ${ }^{14}$ In the present study, the impaired contractile activity persisted in the POI 6-hour groups of the colon, whereas it recovered in the POI 6-hour groups of the small intestine. These are in agreement with the traditional concepts about the recovery time from POI. ${ }^{20}$ The inhibition of GI motility was not affected similarly at each GI segment. Specifically, the small intestinal function normalized first, and then gastric motility returned to normal, whereas the colon was usually the last portion of the GI tract to regain normal motility after surgery. Thus, colonic dysfunction is the most frequent factor that limits the resolution of POI. ${ }^{4,21}$

Among the complex mechanisms and multiple factors that cause POI, accumulating evidence has suggested that inflammation has a key role in the pathophysiology of POI. ${ }^{8,22}$ The inflammation caused by surgical bowel manipulation affects the duration and severity of GI hypomotility during POI, and therefore has great clinical relevance. We have previously demonstrated that inflammation influences the recovery time. ${ }^{15}$ In line with an earlier study, the present data illustrated that the degree of inflammation in POI was 
significantly increased in each segment of the GI tract. It is notable that the colon of the POI groups showed a markedly increased degree of inflammation. It would imply the abundant presence of inflammation particularly in the colon, which may be attributed to the delayed recovery from ileus.

In addition to the degree of inflammation, we also evaluated the expression of calprotectin in POI. Calprotectin is generally expressed in neutrophils and macrophages, especially activated macrophages and monocytes in the acute inflammatory state. ${ }^{23}$ The expression of calprotectin was significantly increased in the small bowel and colon of the POI groups compared to controls. It should be emphasized that the expression of calprotectin was most prominent in the colon in the POI 6-hour groups. These results are in concordance with those of contractile activity, suggesting that the recovery from ileus varied depending on the organ.

To further support the role of inflammation in the pathogenesis of POI, the expression of mast cells in POI according to the organ was evaluated. Our data showed that the expression of mast cells in the colon was significantly increased in the POI 6-hour groups. The contribution of macrophages and mast cells in the inflammatory cascade of POI was demonstrated by previous studies. ${ }^{4,24}$ Activation of resident macrophages in the intestinal muscularis externa results in the release of cytokines and chemokines. The influx of leukocytes starts and leads to accumulation of prostaglandins and nitric oxide that directly inhibit smooth muscle contractility and finally cause intestinal hypomotility in POI. ${ }^{12,25}$ Neutrophil infiltrates have been considered to inhibit local contractility or general motility through the activation of an adrenergic inhibitory pathway. ${ }^{4}$ When the above-mentioned results are combined, we postulate that our results can partly explain the temporal relationship between the degree of inflammation and the recovery period from POI according to the GI tract organs.

A recent study have been performed on intestinal permeability assays in a mouse model of POI and suggested that mast cell activation during abdominal surgery causes epithelial barrier dysfunction and inflammation of the muscularis externa of the bowel. ${ }^{26}$ The authors concluded that the impairment of the epithelial barrier contributes to the pathogenesis of POI. ${ }^{26}$ The process of mast cells involving the disruption of the epithelial barrier is initiated by the release of prestored and de novo-synthesized mediators. Mast cells trigger the release of protease followed by the activation of PAR-2, which finally results in changes in epithelial TJ permeability. ${ }^{27}$ The exact role of mast cells in POI pathogenesis, especially in terms of the epithelial barrier, remains unexplored; however, our data related to PAR-2 expression supported the above mentioned theory. We observed an increased expression of PAR-2 in the POI groups compared to sham, especially in the colon.

PARs are 7 trans-membrane-spanning, G-protein-coupled receptors that are activated by the cleavage of their $\mathrm{N}$-terminal domain by selective proteolytic enzymes. ${ }^{28}$ PARs are strongly expressed throughout the GI tract in several cell types, including mast cells and smooth muscle cells, enterocytes, myenteric neutrons, and endothelial cells, ${ }^{29}$ which are consequently involved in intestinal permeability, visceral sensation, and smooth muscle contractility. ${ }^{29,30}$ The mechanisms of the effect of PAR-2 on intestinal permeability, inflammation, and recovery time from ileus are not yet fully understood. We believe that the marked colonic expression of PAR-2 may suggest a higher expression of PAR-2 in the colon than in the small intestine and stomach, which can be correlated with enhanced initiation and activation of mast cells in the colon. However, we failed to prove the statistical significance of the increased expression of PAR-2 in the present study. This result may be due to the other mediators, such as histamine and cytokines that operate together with PAR-2 during the genesis of POI. Another possible explanation could be that PAR-2 may not be very specific for the initiation of intestinal inflammation. Further experiments are required to identify the exact mechanism of PAR-2 in intestinal inflammation in conjunction to permeability in POI. A group of animals treated with a recognized PAR-2 receptor antagonist would allow a better understanding of the relationship between PAR-2 receptor and mast cell activation in POI.

Gut paracellular permeability is largely determined by alterations of TJ proteins. ${ }^{31}$ Specifically, activated PAR-2 may directly influence cytoskeleton contraction and cause changes in permeability through configurational changes of TJ proteins. ${ }^{31}$

The TJ is composed of membrane-spanning proteins such as claudin, occludin, junctional adhesion molecules, and tricelluin, which form a selective permeable seal in the paracellular space. Impairment of the intestinal TJ barrier, followed by permeation of luminal noxious molecules, can induce a perturbation of the mucosal immune system and inflammation. ${ }^{32}$ Studies have shown that claudins are the key component and backbone of TJs, which are the main determinants of barrier formation and selectivity of paracellular permeability. ${ }^{33}$ Claudins are classified as either barrier forming or pore forming. Among approximately 24 claudin genes, claudin-1 is known as a barrier-forming protein that decreases paracellular permeability. ${ }^{34}$ On the other hand, claudin-2 is a pore-forming protein that increases paracellular permeability through the formation of channels. ${ }^{35}$ Our data showed decreased expression of claudin-1 but increased expression of claudin-2 in the POI groups compared 
to sham. We presumed that our data may suggest the possibility of increased intestinal permeability in the POI group. Based on this assumption, we hypothesized that alterations of paracellular permeability can be an explanation for the delayed resolution of inflammation by exposing the gut to luminal antigen after surgery. However, the results from indirect estimation of junctional proteins are not enough to support the hypothesis of an increased intestinal permeability. It should be elucidated using functional tests with FITC Dextran probe and western blot for the quantification of $\mathrm{TJ}$ proteins expression in a future study.

This study has clinical relevance because it shows temporary changes in inflammation, PAR-2 expression, and paracellular permeability according to the GI tract organ. However, there are important issues that still need to be addressed. Although we tried to assess the changes in the paracellular permeability indirectly by measuring junctional proteins such as claudin- 1 and claudin-2 in POI according to the organ, we did not estimate the degree of permeability directly. Further investigations with direct measurement of the changes of paracellular permeability by using an Ussing chamber or FITC Dextran are warranted. In addition, therapeutic interventions targeted at each step of inflammation will provide further insights into the pathophysiology of POI and new therapeutic prospects.

In conclusion, the recovery time from POI differed according to the GI tract organ. This finding is consistent with that of a previous report. In concordance with the results of contractile activity, our study suggests the possibility that inflammation may also be associated with paracellular permeability at different time points in GI organs. Therefore, increased inflammation and mucosal permeability may play important roles in the different recovery times from postoperative dysmotility. Our findings are valuable in encouraging future studies on the role of inflammation and permeability in the pathophysiology of POI. Macrophages and mast cells initiate and orchestrate the cascade of inflammatory events. Thus, these immune cells seem to be the most interesting targets for the purgative therapy for POI. The cascade of gut permeability may also be relevant to the emerging therapeutic targets for POI.

\section{Supplementary Materials}

Note: To access the supplementary table and figures mentioned in this article, visit the online version of Journal of Neurogastroenterology and Motility at http://www.jnmjournal.org/, and at https:// doi.org/10.5056/jnm17012.
Financial support: This research was supported by Basic Science Research Program through the National Research Foundation of Korea (NRF) funded by the Ministry of Education (Grant No. R1A1A2).

\section{Conflicts of interest: None}

Author contributions: Yoo Jin Lee analyzed and interpreted the data, and wrote a manuscript; Hyojin Park designed, organized, and mediated the present study, and supervised the manuscript; Zahid Hussain and Young Ju Lee performed the experiment and interpretation of the data; and Cheal Wung Huh supported preparing the manuscript including table and figures.

\section{References}

1. Delaney CP, Kehlet H, Senagore AJ, et al. On behalf of the Postoperative Ileus Management Council (PIMC). Postoperative ileus: profiles, risk factors and definitions - a framework for optimizing surgical outcomes in patients undergoing major abdominal and colorectal surgery. Clinical consensus update in general surgery (2006). Available from URL: http:// www.clinicalwebcasts.com/PIMC.htm (accessed 24 November 2017).

2. Collins TC, Daley J, Henderson WH, Khuri SF. Risk factors for prolonged length of stay after major elective surgery. Ann Surg 1999;230:251-259.

3. Finan MA, Barton DP, Fiorica JV, et al. Ileus following gynecologic surgery: management with water-soluble hyperosmolar radiocontrast material. South Med J 1995;88:539-542.

4. Boeckxstaens GE, de Jonge WJ. Neuroimmune mechanisms in postoperative ileus. Gut 2009;58:1300-1311.

5. Iyer S, Saunders WB, Stemkowski S. Economic burden of postoperative ileus associated with colectomy in the United States. J Manag Care Pharm 2009;15:485-494.

6. Millan M, Biondo S, Fraccalvieri D, Frago R, Golda T, Kreisler E. Risk factors for prolonged postoperative ileus after colorectal cancer surgery. World J Surg 2012;36:179-185.

7. Vather R, Bissett IP. Risk factors for the development of prolonged postoperative ileus following elective colorectal surgery. Int J Colorectal Dis 2013;28:1385-1391

8. Kalff JC, Türler A, Schwarz NT, et al. Intra-abdominal activation of a local inflammatory response within the human muscularis externa during laparotomy. Ann Surg 2003;237:301-315.

9. Kehlet H. Postoperative ileus. Gut 2000;47(suppl 4):iv85-iv86; discussion iv87.

10. Kalff JC, Schraut WH, Simmons RL, Bauer AJ. Surgical manipulation of the gut elicits an intestinal muscularis inflammatory response resulting in postsurgical ileus. Ann Surg 1998;228:652-663.

11. Kalff JC, Carlos TM, Schraut WH, Billiar TR, Simmons RL, Bauer AJ. Surgically induced leukocytic infiltrates within the rat intestinal muscularis mediate postoperative ileus. Gastroenterology 1999;117:378-387. 
12. Kalff JC, Schraut WH, Billiar TR, Simmons RL, Bauer AJ. Role of inducible nitric oxide synthase in postoperative intestinal smooth muscle dysfunction in rodents. Gastroenterology 2000;118:316-327.

13. Oliveira HM, Sallam HS, Espana-Tenorio J, et al. Gastric and small bowel ileus after severe burn in rats: the effect of cyclooxygenase-2 inhibitors. Burns 2009;35:1180-1184.

14. Park SJ, Choi EJ, Yoon YH, Park H. The effects of prucalopride on postoperative ileus in guinea pigs. Yonsei Med J 2013;54:845-853.

15. Choi HK, Lee YJ, Lee YH, Park JP, Min K, Park H. Inflammatory responses in the muscle coat of stomach and small bowel in the postoperative ileus model of guinea pig. Yonsei Med J 2013;54:1336-1341.

16. Park HJ, Lee HY, Chung JP, et al. The effects of nitric oxide and vasoactive intestinal peptide on the smooth muscle relaxation of a Guinea pig's gastric fundus. Korean J Gastroenterol 1997;29:289-300.

17. Kim HS, Choi EJ, Park H. The effect of mosapride citrate on proximal and distal colonic motor function in the guinea-pig in vitro. Neurogastroenterol Motil 2008;20:169-176.

18. Tonini M, Galligan JJ, North RA. Effects of cisapride on cholinergic neurotransmission and propulsive motility in the guinea pig ileum. Gastroenterology 1989;96(5 Pt 1):1257-1264.

19. The FO, Bennink RJ, Ankum WM, et al. Intestinal handling-induced mast cell activation and inflammation in human postoperative ileus. Gut 2008;57:33-40.

20. Prasad M, Matthews JB. Deflating postoperative ileus. Gastroenterology 1999;117:489-492.

21. Sunil S, Sinha S. Postoperative ileus: a preventable event (Br J Surg 2000;87:1480-1493). Br J Surg 2001;88:594-595.

22. de Jonge WJ, van den Wijngaard RM, The FO, et al. Postoperative ileus is maintained by intestinal immune infiltrates that activate inhibitory neural pathways in mice. Gastroenterology 2003;125:1137-1147.

23. Bhardwaj RS, Zotz C, Zwadlo-Klarwasser G, et al. The calcium-binding proteins MRP8 and MRP14 form a membrane-associated heterodimer in a subset of monocytes/macrophages present in acute but absent in chronic inflammatory lesions. Eur J Immunol 1992;22:1891-1897.

24. van Bree SH, Nemethova A, Cailotto C, Gomez-Pinilla PJ, Matteoli G, Boeckxstaens GE. New therapeutic strategies for postoperative ileus. Nat Rev Gastroenterol Hepatol 2012;9:675-683.

25. Schwarz NT, KalffJC, Türler A, et al. Prostanoid production via COX-2 as a causative mechanism of rodent postoperative ileus. Gastroenterology 2001;121:1354-1371.

26. Snoek SA, Dhawan S, van Bree SH, et al. Mast cells trigger epithelial barrier dysfunction, bacterial translocation and postoperative ileus in a mouse model. Neurogastroenterol Motil 2012;24:172-184, e191.

27. De Winter BY, van den Wijngaard RM, de Jonge WJ. Intestinal mast cells in gut inflammation and motility disturbances. Biochim Biophys Acta 2012;1822:66-73.

28. Nystedt S, Larsson AK, Aberg H, Sundelin J. The mouse proteinaseactivated receptor- $2 \mathrm{cDNA}$ and gene. Molecular cloning and functional expression. J Biol Chem 1995;270:5950-5955.

29. Sato K, Ninomiya H, Ohkura S, Ozaki H, Nasu T. Impairment of PAR-2-mediated relaxation system in colonic smooth muscle after intestinal inflammation. Br J Pharmacol 2006;148:200-207.

30. Coelho AM, Vergnolle N, Guiard B, Fioramonti J, Bueno L. Proteinases and proteinase-activated receptor 2: a possible role to promote visceral hyperalgesia in rats. Gastroenterology 2002;122:1035-1047.

31. Bueno L, Fioramonti J. Protease-activated receptor 2 and gut permeability: a review. Neurogastroenterol Motil 2008;20:580-587.

32. Lee SH. Intestinal permeability regulation by tight junction: implication on inflammatory bowel diseases. Intest Res 2015;13:11-18.

33. Tamura A, Hayashi H, Imasato M, et al. Loss of claudin-15, but not claudin-2, causes $\mathrm{Na}^{+}$deficiency and glucose malabsorption in mouse small intestine. Gastroenterology 2011;140:913-923.

34. Suzuki T. Regulation of intestinal epithelial permeability by tight junctions. Cell Mol Life Sci 2013;70:631-659.

35. Kotler BM, Kerstetter JE, Insogna KL. Claudins, dietary milk proteins, and intestinal barrier regulation. Nutr Rev 2013;71:60-65. 